\title{
Convexity and Toeplitz Quantization: Kostant's Theorem For The symplectomorphism Group Of The Sphere
}

\author{
Bouleryah, Mohamed Lemine \\ Department of Mathematics \\ King Khalid Universtiy, Abha, February 21, 2019
}

\begin{abstract}
In this paper we use Toeplitz quantization to extend in a very natural way Kostant's theorem for the group $S U(m)$ to the group of symplectomorphisms of the unit sphere and we also give another proof of the infinite dimensional version of Schur and Horn theorem for the sphere based on Schur and Horn theorem for Hermitian matrices.
\end{abstract}

Keywards: Symplectic Geometry,Toeplitz quantization, decreasing rearrangement, mojorization, convexity, Hermitian matrix, measure preserving measure transformations, spectral measure.

\section{Introduction}

This is a continuation of our past work [?, ?] where we have shown certain analogies between infinite dimensional Lie group and finite compact Lie group. In this paper we exhibit close relationships between Kostant's Theorem for the special unitary group and the infinite version of Kostant'Theorem for unit sphere in $\mathbb{R}^{3}$ that was proved in [?].

Kostant's Theorem for $S U(m+1)$ reads: Let $T^{m}$ be the subgroup of diagonal matrices. The Lie algebra of $K^{m}=S U(m+1)$ and $T^{m}$ denoted respectively by $\mathfrak{k}^{m}=u(m+1)$ and $\mathfrak{t}^{m} ; u(m+1)$ consists of all skew Hermitian matrices and $\mathfrak{t}^{m}$ is the set of all purely imaginary diagonal matrices . Let $W^{m}$ denote the Weyl group which in this case coincides with $\sum_{(m+1)}$, the symmetric group.

For $\lambda^{m} \in \mathfrak{t}^{m}$, let $O_{\lambda^{m}}$ denote the orbit $\left\{k \lambda k^{-1} \mid k \in K\right\} \subset u(m+1)$ of skew Hermitian matrices with fixed eigenvalues $\left(\lambda_{j}^{m}\right)_{j}$. Let $\pi_{m}: u(m+1) \rightarrow \mathfrak{t}^{m}$ be the orthogonal projection relative to the inner product on $u(m+1)$ given by minus the trace of the product of two matrices; the effect of $\pi_{m}$ is to pick up the diagonal part of a matrix.

Identify the Lie algebra $u(m+1)$ of skew Hermitian matrices with the vector space of Hermitian matrices via multiplication by $\sqrt{-1}$. 
The theorem states that $\pi_{m}\left(\sqrt{-1} O_{\lambda^{m}}\right)$ is a convex polytope which coincides with the convex hull of the Weyl group orbit $\sqrt{-1} \sum_{(m+1)} \cdot \lambda^{m}$.

Kostant's Theorem for $S U(m+1)$ is the Lie theoretic formulation of Schur and Horn Theorem:

Schur's Theorem: Let $\lambda^{m}=\left(\lambda_{0}^{m}, \cdots, \lambda_{m}^{m}\right) \in \mathbb{R}^{m+1}$ be the eigenvalues of a hermitian matrix $A$ put in a non-increasing order $\lambda_{0}^{m} \geq \cdots \geq \lambda_{m}^{m}$. Let $\left(a_{00}, \cdots, a_{m m}\right)$ be the diagonal of $A$ also ordered in non increasing order $a_{00} \geq$ $\cdots \geq a_{m m}$ then

$$
\sum_{i=0}^{i=k} a_{i i} \leq \sum_{i=0}^{i=k} \lambda_{i}^{m}, \quad 0 \leq k \leq m-1, \quad \sum_{i=0}^{i=m} a_{i i}=\sum_{i=0}^{i=m} \lambda_{i}^{m}
$$

Horn's Theorem: Let $\left(a_{00}, \cdots, a_{m m}\right) \in \mathbb{R}^{m+1}$ such that relations (??) are verified, then there exists a Hermitian matrix $A$ that has $\lambda^{m}$ for spectrum and its main diagonal is $\left(a_{00}, \cdots, a_{m m}\right)$.

In order to find the reformulation of Kostant's theorem for the sphere, we will look at the behaviour of each ingredient in the Kostant's theorem: we will see that a square integrable function $f$ on the sphere can be identified with a sequence $T_{f}^{m}$ of hermitian matrices, that the set of eigenvalues for the matrices $T_{f}^{m}$ determine the decreasing rearrangement of the function $f$, that the union of all maximal tori $T^{m}$ is the group of diffeomorphisms of the sphere that rotates the circles of latitudes. We will also prove that the union of the $\sum_{(m+1)}$ symmetric group of $(m+1)$ letters is nothing but the semi-group of all preserving measure transformations of the unit interval. Our main tools in this work are Toeplitz quantization. We also use material on the pre-ordering majorization and decreasing rearrangement theory. We will review these two notions for the convenience of the reader.

\subsection{Toeplitz Quantization}

Let $\Omega$ be the Fubini-Study Symplectic form on the sphere $\mathbb{C P}(1)$ which is in the local coordinate $[1, w]$ is given by

$$
\Omega=\frac{i}{(1+w \bar{w})^{2}} d w \wedge d \bar{w}
$$

The tensor power $\mathbf{L}^{\otimes m}$ of the standard hyperplane bundle $\mathbf{L}$ has $(m+1)$ linearly independent sections which in local coordinate $w$ are just $1, w, \ldots, w^{m}$. The vector space $\Gamma_{h o l}^{m}$ is spanned by $1, w, \ldots, w^{m}$ and comes equipped with the scalar product

$$
<s_{1}, s_{2}>=\int_{\mathbb{C P}(1)} \frac{1}{\left(1+|w|^{2}\right)^{m}} s_{1}(w) \overline{s_{2}(w)} d \Omega .
$$

Now let $\Gamma_{2}^{m}$ be the space of square-integrable sections of $\mathbf{L}^{\otimes m}$. The orthogonal projection $\Gamma_{2}^{m} \rightarrow \Gamma_{h o l}^{m}$ is denoted by $P^{m}$. 
Definition 1.1 Let $f: \mathbb{C P}(1) \rightarrow \mathbb{R}$ be a square integrable function. The Toeplitz quantization of $f$ is the map $T_{f}^{m}: \Gamma_{\text {hol }}^{m} \rightarrow \Gamma_{\text {hol }}^{m}$ defined by

$$
T_{f}^{m}=P^{m} \circ \mathbf{M}_{f} \circ P^{m},
$$

where $\mathbf{M}_{f}$ is multiplication by $f$.

Proposition 1.1 With the usual coordinates $w=r \exp (i \theta)$, we have:

1. The holomorphic sections $\gamma_{m, k} w^{k}, k=0, \cdots, m$ where $\gamma_{m, k}=\sqrt{\frac{1}{2 \pi}} \sqrt{m+1} \sqrt{\left(\begin{array}{c}m \\ k\end{array}\right)}$ constitute an orthonormal basis of $\Gamma_{h o l}^{2}$ with respect to the inner product (??).

The entries of the matrix $T_{f}^{m}$ in this basis are

$$
\left(T_{f}^{m}\right)_{k, l}=2 \gamma_{m, k} \gamma_{m, l} \int_{0}^{2 \pi} \int_{0}^{+\infty} \frac{f(r, \theta) r^{k+l+1} \exp ((k-l) i \theta) r d r d \theta}{\left(1+r^{2}\right)^{m+2}}
$$

2. Expand $f(r, \theta)$ in a Fourier series:

$$
f(r, \theta)=\sum_{s=-\infty}^{s=+\infty} \hat{f}_{s}(r) \exp (i s \theta)
$$

Let $m, k \rightarrow \infty$ so that $\frac{k}{m}=t$ is fixed and $k-l=s \in \mathbb{Z}$. Then

$$
\left(T_{f}^{m}\right)_{k, l} \rightarrow \hat{f}_{-s}\left(r_{k}^{m}\right), \text { where } r_{k}^{m}=\sqrt{t \backslash(1-t)} .
$$

3. If $f(r \exp (i \theta))=f(r)$, then $T_{f}^{m}$ is a diagonal matrix.

4. $\operatorname{trace}\left(T_{f}^{m}\right)=\frac{(m+1)}{2 \pi} \int_{\mathbb{C P}(1)} f d \Omega$.

\section{Proof:}

1. The hermitian product (??) applied to the sections $w^{k}=r^{k} \exp (i k \theta), w^{l}=r^{l} \exp (i l \theta)$ gives

$$
\left\langle w^{k}, w^{k}\right\rangle=4 \pi \int_{0}^{+\infty} \frac{r^{2 k+1} r d r}{\left(1+r^{2}\right)^{m+2}}=\frac{1}{\gamma_{m, k}}
$$

and a table of integrals gives the value stated of $\gamma_{m, k}$.

2. We also get the entries of the matrix $T_{f}^{m}$ :

$$
\begin{aligned}
\left\langle f w^{k}, w^{l}\right\rangle & =\gamma_{m, k} \gamma_{m, l} \int_{\mathbb{C P}(1)} h_{m}\left(f z^{k}, z^{l}\right) d \Omega \\
& =\gamma_{m, k} \gamma_{m, l} \int_{0}^{2 \pi} \int_{0}^{\infty} \frac{f(r, \theta) r^{k+l+1} \exp ((k-l) i \theta) 2 r d r d \theta}{\left(1+r^{2}\right)^{m+2}} \\
& \left.=4 \pi \gamma_{m, k} \gamma_{m, l} \int_{0}^{\infty} \frac{r^{k+l+1}}{\left(1+r^{2}\right)^{m+2}} \widehat{f(r, \theta)}\right)_{l-k} d r
\end{aligned}
$$


3. Let us rewrite the entry $\left(T_{f}^{m}\right)_{k, l}$ in the form

$$
\left(T_{f}^{m}\right)_{k, l}=2 \gamma_{m, k} \gamma_{m, l} \int_{0}^{\infty} \frac{r^{l-k+1} \int_{0}^{2 \pi} f(r, \theta) e^{(k-l) i \theta} d \theta}{\left(1+r^{2}\right)^{2}} \exp \left(-m \phi\left(r, \frac{2 k}{m}\right)\right) d r
$$

where

$$
\phi\left(r, \frac{2 k}{m}\right)=\ln \left(1+r^{2}\right)-\frac{2 k}{m} \ln r .
$$

The asymptotic approximation of $\left(T_{f}^{m}\right)_{k, l}$ is a straight forward application of Laplace approximation

to $\gamma_{m, k}, \gamma_{m, l}$ and to $\int_{0}^{\infty} \frac{r^{l-k+1} \int_{0}^{2 \pi} f(r, \theta) e^{(k-l) i \theta} d \theta}{\left(1+r^{2}\right)^{2}} \exp \left(-m \phi\left(r, \frac{2 k}{m}\right)\right) d r$.

Laplace approximation reads

$$
\int_{0}^{\infty} h(x) e^{-m \phi(x)} d x=h\left(x_{0}\right) e^{-m \phi\left(x_{0}\right)} \sqrt{\frac{2 \pi}{m \phi^{\prime \prime}\left(x_{0}\right)}}+O\left(\frac{1}{m}\right)
$$

where the function $\phi$ has a local minimum at $x_{0}$.

4. If $f(r, \theta)=f(r)$,

$$
\left(T_{f}^{m}\right)_{k, l}=4 \pi \gamma_{m, k} \gamma_{m, l} \int_{0}^{\infty} \frac{f(r) r^{l+k+1} d r}{\left(1+r^{2}\right)^{m+2}} \delta_{k}^{l} .
$$

where $\delta_{k}^{l}$ is the Kronecker symbol.

5.

$$
\begin{aligned}
\operatorname{tr}\left(T_{f}^{m}\right) & =\sum_{k=0}^{k=m} \gamma_{m, k}^{2} \int_{0}^{2 \pi} \int_{0}^{\infty} \frac{f(r, \theta) r^{2 k+1} 2 d r d \theta}{\left(1+r^{2}\right)^{m+2}} \\
& =\sum_{k=0}^{k=m} \frac{1}{2 \pi}(m+1)\left(\begin{array}{c}
m \\
k
\end{array}\right) \int_{0}^{2 \pi} \int_{0}^{\infty} \frac{f(r, \theta) r^{2 k} 2 r d r d \theta}{\left(1+r^{2}\right)^{m+2}} \\
& =(m+1) \frac{1}{2 \pi} \int_{\mathbb{C P}(1)} f d \Omega .
\end{aligned}
$$

\section{$2 \quad \lim _{m \rightarrow+\infty} s u(m+1)$}

Toeplitz quantization allows us to associate to a function $f \in L^{2}(\mathbb{C P}(1), \Omega)$ a Hermitian matrix $T_{f}^{m}$ and conversely it is proved in [?] proposition (4.2) that the map $f \longmapsto T_{f}^{m}$ is surjective. Also we have seen that asymptotically $\left(T_{f}^{m}\right)_{k, l}$ is the Fourier coefficient $\hat{f}_{l-k}(r)$ of the function $f$.

Since matrices and functions are not from the nature, it will be more convenient to identify the vector space of Hermitian matrices with a subspace of step functions of $L^{2}(\mathbb{C P}(1), \Omega)$. To do so let $\phi$ be the local chart defined on the sphere 
minus the North pole by

$$
\begin{gathered}
\mathbb{C P}(1) \backslash\{\text { North pole }\} \longrightarrow[0,1] \times[0,1] \\
\phi(r, \theta)=\left(\frac{1}{1+r^{2}}, \theta\right) .
\end{gathered}
$$

Let $I_{k, l}^{m}=\left[\frac{k}{m+1}, \frac{k+1}{m+1}\left[\times\left[\frac{l}{m+1}, \frac{l+1}{m+1}\left[\right.\right.\right.\right.$ and let $S_{k, l}^{m}=\phi^{-1}\left(I_{k, l}^{m}\right)$.

To the Hermitian matrix $A=\left(a_{k l}\right)_{k, l}$, we associate the step function

$$
S_{A}(r, \theta)=a_{k, l},(r, \theta) \in S_{k, l}^{m} .
$$

In this way the vector space $\mathcal{H}_{m}$ of $m \times m$ Hermitian matrices is identified with a subspace of $L^{2}(\mathbb{C P}(1), \Omega)$. These identifications allows one to think of $s u(\infty)$ as all square integrable function on the sphere $\mathbb{C P}(1)$.

\section{Maximal torus}

Theorem 3.1 Let $\mathcal{T}(\mathbb{C P}(1))$ be the group of all invertible measure transformations of the sphere that rotate every circle of latitude. The torus $T^{m}$ can be identified with a subgroup of $\mathcal{T}(\mathbb{C P}(1))$ and therefore $\mathcal{T}(\mathbb{C P}(1))$ is union of all the tori $T^{m}$.

Proof:

Think of $\mathbb{C P}(1)$ as the sphere in $\mathbb{R}^{3}$. Then the one dimensional torus $S^{1}$ acts on it by rigid rotations about the vertical axis. This action is Hamiltonian with respect to the Fubini-Study Symplectic form $\Omega$ and has a moment map $J: \mathbb{C P}(1) \longrightarrow \mathbb{R}$ that associates to each point its height above the equatorial plane:

$$
J: \mathbb{C P}(1) \longmapsto[0,1] ;\left[1, r e^{i \theta}\right] \longmapsto \frac{1}{1+r^{2}} .
$$

For $I_{k}^{m}=\left[\frac{k}{m+1}, \frac{k+1}{m+1}\left[, k=0,1, \cdots, m\right.\right.$, let $S_{k}^{m}=J^{-1}\left(I_{k}^{m}\right)$ be a spherical segment. Let $\left(e^{i \theta_{0}}, e^{i \theta_{1}}, \ldots, e^{i \theta_{m}}\right)$ be an element of the torus $T^{m}=S^{1} \times S^{1} \times \ldots \times S^{1}$.

$T^{m}$ acts on $\mathbb{C P}(1)$ by rotating each spherical segment $S_{k}^{m}$ by an angle $\theta_{k}$ around the vertical axis.

Of course, this action is not differentiable if the angles $\theta_{k}$ are different. But these spherical segments get thinner and thinner as $m$ gets bigger and bigger. Ultimately, they will shrink down to circles and the action of the tori $T^{m}$ will be just rotating the circles of latitudes.

So for each $m$ we will identify the torus $T^{m}$ with the subgroup of $\mathcal{T}(\mathbb{C P}(1))$. Let $\left(\theta_{0}, \theta_{1}, \ldots, \theta_{m}\right) \in \mathbb{R}^{m+1}$ and let $\chi_{k}$ be the characteristic function of the interval $I_{k}=\left[\frac{k}{m+1}, \frac{k+1}{m+1}\left[\right.\right.$. Let $f_{m}(r)=\sum_{k=0}^{k=m} \theta_{k} \chi_{k}(r)$.

The action of the torus $T^{m}$ on the sphere can be written

$$
\left(e^{i \theta_{0}}, e^{i \theta_{1}}, \ldots, e^{i \theta_{m}}\right) \cdot[1, z]=e^{i f_{m}(r)} \cdot[1, z]=\left[1, e^{i f_{m}(r)} z\right] .
$$


Conversely let $\phi \in \mathcal{T}(\mathbb{C P}(1))$. In the chart $\left[1, r e^{i \theta}\right]$, we can write $\phi(r, \theta)=$ $\left(r, e^{i \theta+i f(r)}\right)$ for a certain measurable function $f$.

Since the function $f$ can be approximated by a sequence of step functions $f_{m}(r)=\sum_{k=0}^{k=m} \theta_{k} \chi_{k}$ such that the sequence $\left(e^{i f_{m}} \cdot[1, w]\right)_{m}$ converges to $\left(e^{i f}\right.$. $[1, w]$, we conclude that $\phi \in \lim \sup T^{m}$.

It is interesting to notice the one time flow of the hamiltonian vector field $X_{f}$ of a function $f$ that is constant on the circles of latitudes rotates the circles of latitudes. In other words, $\operatorname{Exp}\left(X_{f}\right)$ is just our usual exponential of lie group. We have the following commutative diagram where $\operatorname{Smeas}(\mathbb{C P}(1)$ is the set of all invertible measure preserving transformations of the sphere.

i $S^{m}$ is sending $\left(\theta_{0}, \theta_{1}, \cdots, \theta_{m}\right)$ to the step function that equal to $\theta_{k}$ on the spherical segment $S_{k}^{m}$.

ii $\operatorname{Rot}^{m}$ is the map that rotates each spherical segment with angle $\theta_{k}$.

iii $\exp$ is the usual exponent $\left(\theta_{0}, \theta_{1}, \cdots, \theta_{m}\right) \rightarrow\left(e^{i \theta_{0}}, e^{i \theta_{1}}, \cdots, e^{i \theta_{m}}\right)$.

iiii $\operatorname{EXP}(f)$ is the rotation with an angle of $f(r)$.

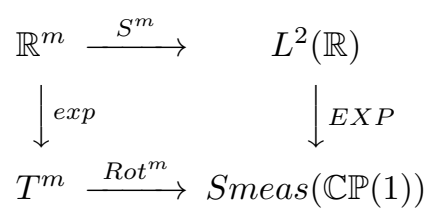

\section{Eigenvalues}

Before looking at the asymptotic behaviour of the eigenvalues of the hermitian matrix $T_{f}^{m}$, let us review quickly the notion of decreasing rearrangement of a function. This is a deep generalization of the simple act of arranging a finite list of numbers in decreasing order. A function is a list of continuously many numbers, and it may be useful, in certain applications, to rearrange those in a different order, for instance because comparisons between complicated functions become feasible.

Let $f:(X, \mu) \rightarrow \mathbb{R}$ be a measurable function. Let $d_{f}(t)=\mu(\{\omega: f(\omega) \geq t\})$ be the distribution function of $f$.

The decreasing rearrangement $f^{*}$ of the function $f$ is

$$
f^{*}(x)=\inf \left\{t: d_{f}(t)<x\right\}=\sup \left\{t: d_{f}(t)>x\right\}, \quad 0 \leq x \leq 1
$$

Because of the importance of $f^{*}$ in the sequel, we will follow [?, ?] and describe how to construct the decreasing rearrangement of a step function:

Example 4.1 let $f(x)=\sum_{j} u_{j} \chi_{E_{j}}(x)$, where $\left\{E_{j}\right\}$ is a partition of $[0,1]$. We order the values of $f$ in a decreasing order: 
$t_{1}>t_{2}>\cdots>t_{p}$ and to each values $t_{j}$ we associate the set $F_{j}=f^{-1}\left(t_{j}\right)$.

Let

$$
a_{0}=0, a_{1}=\left|F_{1}\right|, a_{2}=\left|F_{1}\right|+\left|F_{2}\right|, \cdots a_{p}=\sum_{1}^{p}\left|F_{k}\right|=1
$$

With $t_{1}=\max f(x)_{x \in[0,1]}, \quad t_{p}=\min f(x)_{x \in[0,1]}$, we get

$$
f^{*}(s)=\left\{\begin{array}{cccc}
t_{1} & 0 \leq & s & <a_{1} \\
t_{2} & a_{1} \leq & s & <a_{2} \\
\vdots & \vdots & \ldots & <\vdots \\
t_{p} & a_{p-1} \leq & s & \leq 1
\end{array}\right.
$$

Fore more on decreasing rearrangement of a function, one can also see [?].It a striking fact that the decreasing rearrangement o the function $f$ is determined by the decreasing rearrangement of the eigenvalues of the matrix $T_{f}^{m}$ as it is shown by the following theorem.

Theorem 4.1 Let $T_{f}^{m}$ be the Toeplitz quantization of $f \in C^{\infty}(\mathbb{C P}(1))$ and let $\lambda^{m}=\left(\lambda_{0}^{m}, \lambda_{1}^{m}, \cdots, \lambda_{m}^{m}\right)$ be the eigenvalues of $T_{f}^{m}$ arranged in non-increasing order.

Let $\Lambda^{m}(r)$ the real step function defined on the interval $[0,1[$ by

$$
\Lambda^{m}\left(\left[\frac{k}{m+1}, \frac{k+1}{m+1}[)=\lambda_{k}^{m}, \quad 0 \leq k \leq m\right.\right.
$$

Then the sequence of decreasing rearrangement of the functions $\Lambda^{m}(r)$ converges point-wise almost everywhere to the decreasing rearrangement $f^{*}$ of the function $f$.

\section{Proof :}

In [?], V.Guillemin has proved in a more general setting a version of Szegö' Theorem ,that in the case of our sphere says: : for every continuous function $\phi$,

$$
\lim _{m \mapsto+\infty} \sum_{k=0}^{k=m} \frac{1}{m+1} \phi\left(\lambda_{k}^{m}\right)=\frac{1}{2 \pi} \int_{\mathbb{C P}(1)} \phi(f) d \Omega
$$

But since $f$ and $f^{*}$ are equi-measurable, we have

$$
\frac{1}{2 \pi} \int_{\mathbb{C P}(1)} \phi(f) d \Omega=\frac{1}{2 \pi} \int_{0}^{1} \phi \circ f^{*}(t) d t .
$$

Relation (??) becomes

$$
\begin{aligned}
\lim _{m \mapsto+\infty} \sum_{k=0}^{k=m} \frac{1}{m+1} \phi\left(\lambda_{k}^{m}\right) & =\int_{0}^{1} \phi \circ f^{*}(t) d t \\
\lim _{m \mapsto+\infty} \int_{0}^{1} \phi\left(\Lambda^{m}\right)(t) d t & =\int_{0}^{1} \phi\left(f^{*}\right)(t) d t
\end{aligned}
$$


Relation ( ?? ) is equivalent to: the sequence of step functions $\left(\Lambda^{m}\right)_{m}$ converges in distribution to the real function $f^{*}$. ( [ [?],p. 84] is a good reference for more details on convergence in distribution.)

A well known theorem by Skorokhod ( [?]) states that sequence of the decreasing rearrangement of $\left(\Lambda^{m}\right)_{m}$ converge point-wise to the decreasing rearrangement of $\left(f^{*}\right)$. But since $f^{*}$ and $\Lambda^{m}$ are decreasing functions, we have $\left(f^{*}\right)^{*}=f^{*}$, and $\left(\Lambda^{m}\right)^{*}=\Lambda^{m}$.

Consequently, the sequence $\left(\Lambda^{m}\right)_{m}$ converges point-wise almost everywhere to $f^{*}$.

Example 4.2 Let $J(w)=J(r \exp (i \theta))=\frac{1}{1+r^{2}}$. Because $J$ depends only on $r, T_{J}^{m}$ is a diagonal matrix and its spectrum is:

$$
\begin{aligned}
\left(T_{J}^{m}\right)_{k, k}=\lambda_{k}^{m} & =\left(\gamma_{m, k}\right)^{2} 4 \pi \int_{0}^{+\infty} \frac{r^{2 k+1} r d r}{\left(1+r^{2}\right)^{m+3}} \\
& =\frac{4 \pi\left(\gamma_{m, k}\right)^{2}}{4 \pi\left(\gamma_{m+1, k}\right)^{2}} \\
& =\frac{\frac{1}{2 \pi}(m+1)\left(\begin{array}{c}
m \\
k
\end{array}\right)}{\frac{1}{2 \pi}(m+2)\left(\begin{array}{c}
m+1 \\
k
\end{array}\right)} \\
& =\frac{m+1-k}{m+2}
\end{aligned}
$$

and if we set $s=k \backslash m$, in (??), then

$$
\Lambda^{m}(s)=\lambda_{k}^{m}=\frac{1+\frac{1}{m}-s}{1+\frac{2}{m}}
$$

from which we get

$\lim _{m \mapsto+\infty} \Lambda^{m}(s)=1-s$ or $J^{*}(s)=1-s$.

\section{$5 \quad$ Weyl group}

In this section we will show that when $m$ goes to infinity, the symmetric group $\sum_{m}$ can be identified with the set of measure preserving transformation of the unit interval.

The Lebesgue measure on the unit interval $I=[0,1]$ will be always denoted by $|\cdot|$.

A map $\phi$ from $[0,1]$ to itself is a measure preserving transformation if

$$
\left|\phi^{-1}(A)\right|=|A|, \quad \text { for Borel set } \mathrm{A}
$$

The set of all (non necessary invertible ) measure preserving transformation of the unit interval will be denoted $S m e a s(I)$. The invertible ones will be denoted by Imeas(I). Each $S \in \operatorname{Smeas}(I)$ determine a bounded linear operator $P_{S}$ on 
$L^{2}([0,1])$ by $P_{S}(f)=f \circ S$. In this way, Smeas $(I)$ can be identified to a subset of the set of bounded linear operators of $\left.L^{2}[0,1]\right)$ and the strong operator topology induces a topology on $\operatorname{Smeas}(I)$.

Evidently, a sequence $S_{n}$ converges to $S$ in the strong operator topology if for every function $f, f \circ S_{n}$ converges to $f \circ S$ in $L^{2}([0,1])$.

Let $I_{k}^{m}=[k \backslash(m+1),(k+1) \backslash(m+1)), k=0,1, \cdots, m ; m=0,1, \cdots$.

Let $\sum_{m}$ be the group of permutations of $(m+1)$ letters. For $\sigma \in \sum_{m}, \widehat{\sigma}$ is the invertible measure preserving transformation that sends the interval $I_{k}^{m}$ to the interval $I_{\sigma(k)}^{m}$ by ordinary translation.

We call $\hat{\sigma}$ a permutation of rank $m$. In this way, we can identified the group of permutations $\sum_{m}$ with a subgroup of Imeas(I).

Halmos ([?]) shows that the set of (dyadic) permutations of different rank is dense in Imeas(I) for the strong operator topology.

Also Brown, in [?] has proved that $\operatorname{Smeas}(I)$ is the closure of $\operatorname{Imeas}(I)$ for the strong operator topology.

We can summarize these results in the following theorem.

Theorem 5.1 Let $\sum_{m}$ be the symmetric group of $(m+1)$ letters. There exists a one to one group homomorphism $\Psi^{m}: \sum_{m} \longrightarrow$ Imeas(I) that sends $\sigma$ to $\widehat{\sigma}$ and if we identify $\sum_{m}$ with $\Psi^{m}\left(\sum_{m}\right)$, then $\bigcup_{m}^{m} \sum_{m}$ is a dense set in Smeas(I) for the strong operator topology.

So the Weyl group in Kostant's theorem for $\mathrm{SU}(\mathrm{m}+1)$ witch is the symmetric group of $(\mathrm{m}+1)$ letters becomes the semigroup Smeas(I), the set of non-invertible measure transformations of the unit interval.

\section{The Orbit of the Weyl group}

We would like to see here the behaviour of the Weyl group orbit $\sum_{m}\left(\lambda^{m}\right)$ when $m$ gets bigger. We have seen that the group of permutations $\sum_{m}^{m}$ can be identified with the subgroup of invertible measure transformation of the unit interval $[0,1]$ and also that the eigenvalues $\lambda_{k}^{m}$ determine the decreasing rearrangement of the function $f$. Therefore it reasonable to expect that the orbit of the Weyl group $\sum_{m}\left(\lambda^{m}\right)$ will be

$\operatorname{Smes}(I) \cdot f^{*}=\left\{f^{*} \circ \phi, \mid \phi\right.$ is a measure preserving transformation of $[0,1]$.

To be more precise, let us identify the spectrum $\lambda^{m}=\left(\lambda_{m}^{0}, \lambda_{m}^{1}, \cdots, \lambda_{m}^{m}\right)$ of the matrix $T_{f}^{m}$ with the step function $\Lambda^{m}$ defined on the unit interval $[0,1]$ by $\Lambda^{m}(t)=\lambda_{m}^{k}, t \in\left[\frac{k}{m+1}, \frac{k+1}{m+1}[\right.$.

The orbit $\sum_{m} \cdot \lambda^{m}$ is therefore identified with the set of step functions $\mathcal{O}\left(\Lambda^{m}\right)=$ $\left\{\Lambda^{m} \circ \widehat{\sigma}, \mid \widehat{\sigma}\right.$ a permuation of the intervals $\left[\frac{k}{m+1}, \frac{k+1}{m+1}[\}\right.$.

Bearing these identifications in mind we claim that the topological limit sup of $\mathcal{O}\left(\Lambda^{m}\right)$ is $\operatorname{Smes}(I) \cdot f^{*}$.

Recall that if $\left\{E_{m}\right\}$ is a sequence of subsets of a topological space X, a point $\mathrm{x}$ in $\mathrm{X}$ belongs to the topological $\lim \sup$ of $E_{m}$, denoted $L s E_{m}$, if for every 
neighborhood $\mathrm{V}$ of $\mathrm{x}$ there are infinitely many $\mathrm{m}$ with

$V \cap E_{m} \neq \emptyset$. We can also characterized elements of $L s E_{m}$ by the existence of a generalized sequence that converges to this element.

Proof : We want to show $\operatorname{Ls} \mathcal{O}\left(\Lambda^{m}\right)=\operatorname{Smes}(I) \cdot f^{*}$.

Let $f^{*} \circ \phi \in \operatorname{Smeas}(I) \cdot f^{*}$.

The set of (dyadic) permutations is from theorem (??) dense in $\operatorname{Smeas}(I)$ for the strong operator topology; therefore there exists a generalized sequence $\left(\sigma_{\alpha}\right)_{\alpha}$ of permutations that converges to $\phi$, i.e for each $m, \Lambda^{m} \circ \sigma_{\alpha}$ converges in $L^{2}([0,1]$ to the function $\Lambda^{m} \circ \phi$. Also since the sequence $\left(\Lambda^{m}\right)$ converges almost everywhere to $f^{*}$ we see that we can extract a generalized subsequence $\left(\Lambda^{k} \circ \sigma_{\alpha_{k}}\right)_{k}$ that converges to $f^{*} \circ \phi$. and therefore $f^{*} \circ \phi \in L s \mathcal{O}\left(\Lambda^{m}\right)$.

To prove the converse, let $g \in L s \mathcal{O}\left(\Lambda^{m}\right)$. There exists then a generalized sequence $g_{\alpha}=\Lambda^{m_{\alpha}} \circ \sigma_{\alpha}$ that converges to the function $g$ in $L^{2}([0,1])$.

Each invertible measure preserving transformation $\sigma_{\alpha}$ induces a unitary operator $P_{\sigma_{\alpha}}$ on the Hilbert space $L^{2}([0,1])$ defined by $P_{\sigma_{\alpha}}(h)=h \circ \sigma_{\alpha}$. Since the norm of $P_{\sigma_{\alpha}}$ is equal to 1 , the generalized sequence $\left(\sigma_{\alpha}\right)_{\alpha}$ belongs to the closed sphere of linear operators on $L^{2}([0,1])$.

It is proved in [[?], p.512] that the closed sphere of linear operators on $L^{2}([0,1])$ is a compact set for the weak operator topology, therefore the generalized sequence $P_{\sigma_{n}}$ admits a generalized subsequence, that we denote again $P_{\sigma_{n}}$, that converges weakly to a linear operator $P$. But since the operators $P_{\sigma_{n}}$ are unitary operators, weak convergence and strong convergence are the same.

Also theorem 3 of J.Brown in [[?], p.22] states that the strong closure of the set of invertible measure preserving transformations of the interval $[0,1]$ is the set of all measure preserving transformations of the interval $[0,1]$.

We conclude then that the generalized sequence $\left(\sigma_{\alpha}\right)_{\alpha}$ converges strongly to a measure preserving transformation $\phi$ of the unit interval $[0,1]$.

Now since the sequence $\left(\Lambda^{m}\right)_{m}$ converges to $f^{*}$, it follows that $g_{\alpha}=\Lambda^{m_{\alpha}} \circ \sigma_{\alpha}$ converges to $f^{*} \circ \phi$ and so $g=f^{*} \circ \phi$.

In conclusion, the sequence of the Weyl orbit $\sum_{m} \cdot \Lambda_{m}$ "converges" to the orbit of $f^{*}$ under the action of the semi-group $S$ meas $(I)$ of the set of all measure preserving transformations of $[0,1]$.

\section{Projection}

Recall that the projection $\pi_{m}$ in Kostant's theorem picks up the diagonal of the matrix. To find therefore the limit of $\pi_{m}$ when $m$ goes to infinity we have to find the asymptotic approximation of the main diagonal of the Hermitian matrix $T_{f}^{m}$.

In (?? ), we have shown that $\left(T_{f}^{m}\right)_{k, k}=\frac{1}{2 \pi} \int_{0}^{2 \pi} f(r, \theta) d \theta+O\left(\frac{1}{m}\right)$. Also the identification of a Hermitian matrix with step function that we defined in (??) allows us to identify the diagonal of $T_{f}^{m}$ with the step function $\sum_{k}\left(T_{f}^{m}\right)_{k, k} S_{k, k}^{m}$ that converges to $\frac{1}{2 \pi} \int_{0}^{2 \pi} f(r, \theta) d \theta$. 
For simplicity, we write

$$
\begin{aligned}
\lim _{m \longrightarrow \infty} \pi_{m}\left(\left(T_{f}^{m}\right)_{k, l}\right) & =\lim _{m \longrightarrow \infty}\left(T_{f}^{m}\right)_{k, k} \\
& =\frac{1}{2 \pi} \int_{0}^{2 \pi} f(r, \theta) d \theta
\end{aligned}
$$

\section{Orbit}

For $\lambda^{m} \in \mathfrak{t}^{m}$, let $O_{\lambda^{m}}$ denote the set of skew Hermitian matrices with fixed eigenvalues $\left(\lambda_{j}^{m}\right)_{j}$.

For $f \in \in L^{2}(\mathbb{C P}(1)$, the orbit of $f$ is simply be the set of all function $g$ with prescribed decreasing rearrangement $f^{*}$. The orbit $\mathcal{O}(f)$ can also be characterised by the set of functions $g$ such that the matrix $T_{g}^{m}$ and $T_{f}^{m}$ are asymptotically equivalent.

Recall that two sequences of hermitian matrices $A_{m}$ and $B_{m}$ with eigenvalues respectively $\lambda_{m}^{k}$ and $\mu_{m}^{k}$ are asymptotically equivalent if the eigenvalues $\lambda_{m}^{k}$ and $\mu_{m}^{k}$ are bounded and if

$$
\forall p \in \mathbb{N}, \lim _{m \rightarrow \infty} \frac{1}{m+1} \sum_{k=0}^{k=m}\left(\lambda_{m}^{k}\right)^{p}=\lim _{m \rightarrow \infty} \frac{1}{m+1} \sum_{k=0}^{k=m}\left(\mu_{m}^{k}\right)^{p} .
$$

Let us add here that the orbit of $f$ is weakly compact. Indeed according to [?], if $g^{*}=f^{*}$, then there exists a measure preserving transformation $\phi: \mathbb{C P}(1) \rightarrow$ $[0,1]$ such that $g=f^{*} \circ \phi$

Let $S M e a(\mathbb{C P}(1),[0,1]$ be the set of all measure preserving transformations (not necessarily invertible) from $\mathbb{C P}(1)$ to $[0,1]$.

Each $\phi \in S M e a\left(\mathbb{C P}(1),[0,1]\right.$ induces a linear operator $\left.P_{\phi}: L^{2}([0,1]) \rightarrow L^{2}(C P(1), \Omega)\right)$

defined by $P_{\phi}(h)=h \circ \phi$. So the weak operator topology induces a topology on SMea $(\mathbb{C P}(1),[0,1]$.

Since $P_{\phi}$ has norm 1 , and because the unit sphere of linear operator is weakly compact, we conclude from Theorem 3 of J.Brown in [[?], p.22], that the orbit of $f$ is weakly compact.

\section{Kostant'sTheorem}

From all above, we see that the following theorem proved in ([?]) is in fact a straightforward generalization of the Kostant's theorem for the Lie group $S U(m+1)$ : one can think of the function $f$ as a hermitian matrix whose spectrum is $f^{*}$, the orbit of $f$ is then all functions whose decreasing rearrangement is $f^{*}$, the "Weyl group" is in this case the semi-group of all measure preserving transformations of the unit interval $[0,1]$, the projection of $f$ is the zero fourier coefficient of $f$.

Theorem 9.1 Let $f: \mathbb{C P}(1) \longrightarrow \mathbb{R}$ and let $f^{*}$ be the decreasing rearrangement of $f$. 
Let $\mathcal{O}(f)=\left\{g \in L^{2}(\mathbb{C P}(1)), \mid g^{*}=f^{*}\right\}$. Let $\pi: L^{2}(\mathbb{C P}(1)) \rightarrow L^{2}(\mathbb{R})$ that sends $f(r, \theta)$ to through $f^{*}$ of the semi group $W=\{\phi:[0.1] \longrightarrow[0,1], \phi$ is a measure preserving transformation $\}$.

Before giving the proof of the theorem, let us introduce another practical way to characterise convex sets in $\mathbb{R}^{n}$ and in $L^{1}([0,1])$.

\section{Definition 9.1}

1. For $x \in \mathbb{R}^{n}$, let $x^{*}$ denote the vector obtained by rearranging the components of $x$ in non-increasing order.

Then if $x$ and $y$ are vectors in $\mathbb{R}^{n}$ then We say $y$ majorizes $x$ if

$$
\begin{aligned}
x_{1}^{*}+x_{2}^{*}+\cdots x_{k}^{*} & \leq y_{1}^{*}+y_{2}^{*}+\cdots y_{k}^{*}, \cdots \text { for } 1 \leq k \leq n-1, \\
\sum_{1}^{n} x_{k}^{*} & =\sum_{1}^{n} y_{k}^{*}
\end{aligned}
$$

2. Let $f, g \in L^{1}([0,1])$. Let $f^{*}$ and $g^{*}$ be the decreasing rearrangement of $f$ and $g$.

We say that $f$ majorizes $g$ ( written $g \prec f$ ) if

$$
\begin{aligned}
& \int_{0}^{s} g^{*}(z) d z \leq \int_{0}^{s} f^{*}(z) d z, \quad 0 \leq s<1 \\
& \int_{0}^{1} g^{*}(z) d z=\int_{0}^{1} f^{*}(z) d z
\end{aligned}
$$

Remark 9.1 In fact, majorization on $L^{1}([0,1])$ is a generalization of majorization on $\mathbb{R}^{m+1} n$. To see that for $x \in \mathbb{R}^{m+1}$, let $S^{m}(x)$ be the step function defined on $[0,1]$ by

$S^{m}(x)(t)=x_{k}, t \in[k \backslash(m+1),(k+1) \backslash(m+1)[$.

Then if $x$ and $y$ are vectors in $\mathbb{R}^{m+1}$ then $S^{m}(y)$ majorizes $S^{m}(x)$ if

$$
\begin{aligned}
x_{0}^{*}+x_{1}^{*}+\cdots x_{k}^{*} & \leq y_{0}^{*}+y_{1}^{*}+\cdots y_{k}^{*}, \cdots \text { for } 0 \leq k \leq m-1, \\
\sum_{0}^{m} x_{k}^{*} & =\sum_{0}^{m} y_{k}^{*} \\
x & \prec y \Rightarrow S^{m}(x) \prec S^{m}(y)
\end{aligned}
$$

Let $\lambda^{m}=\left(\lambda_{0}^{m}, \lambda_{1}^{m}, \cdots, \lambda_{m}^{m}\right)$ be the spectrum of a Hermitian matrix $A=\left(a_{k l}\right)$. then Schur's theorem says the step function $S^{m}\left(a_{00}, a_{11}, \cdots, a_{m m}\right)$ is majorized by the step function $S^{m}\left(\lambda^{m}\right)$

$$
S^{m}\left(a_{00}, a_{11}, \cdots, a_{m m}\right) \prec S^{m}\left(\lambda^{m}\right) .
$$

We need the following crucial results:

Rado theorem: Let $\lambda^{m}=\left(\lambda_{0}^{m}, \lambda_{1}^{m}, \cdots, \lambda_{m}^{m}\right)$. Let $\operatorname{con}\left(\sum_{m} \cdot \lambda^{m}\right)$ be he convex 
hull of the set of points $\left(\lambda_{\sigma(0)}^{m}, \lambda_{\sigma(1)}^{m}, \cdots, \lambda_{\sigma(m)}^{m}\right)$ where $\sigma$ is a permutation of $(m+1)$ letters. Then

$$
x \prec \lambda^{m} \Leftrightarrow x \in \operatorname{con}\left(\sum_{m} \cdot \lambda^{m}\right)
$$

. Ryff'sTheorem: The set $\Omega(f)=\left\{g \in L^{2}([0,1]) \mid g \prec f\right\}$ is weakly compact and convex. Its set of extreme points is $\{f \circ \phi \mid \phi$ measure preserving transformations of the unit interval $[0,1]\}$.

For the proof of the theorem let us keep in mind the following dictionary:

$$
\begin{gathered}
\text { Hermitian matrix } \\
\text { Torus } T^{m} \\
T_{f}^{m} \\
\text { diagonal matrix } \\
\text { Eigenvalues }\left(\lambda^{m}\right)
\end{gathered}
$$

Orbit of $\left(\lambda^{m}\right)=O_{\lambda^{m}}=$ matrices whose spectrum is $\lambda^{m}$

$$
\begin{gathered}
\text { Weyl group of } s u(m+1)= \\
\sum_{m} \text { :symmetric group } \\
\begin{array}{c}
\sum_{m} \cdot \lambda^{m} \text { :orbit of the Weyl group } \\
\prec \text { :majorization in } \mathbb{R}^{m}
\end{array} \\
\pi_{m}: \text { projection on the diagonal }
\end{gathered}
$$

$$
L^{2}(\mathbb{C P}(1), \Omega)
$$

measure preserving transformations of the sphere that rotates every circle of latitude

$$
f \in L^{2}(\mathbb{C P}(1), \Omega)
$$

functions $f(r, \theta)=f(r)$

dereasing rearrangement of the function $f$

Orbit of $f=O_{f}=$ functions

whose decreasing rearrangement is $f^{*}$

Weyl group of $L^{2}(\mathbb{C P}(1), \Omega)$ : Smeas $(\mathrm{I})=$

all measure preserving transformations of $[0,1]$

$\operatorname{Smeas}(I) \cdot f^{*}=\left\{f^{*} \circ \phi, \phi \in \operatorname{Smeas}(I)\right\}$

$\prec$ majorization in $L^{2}(\mathbb{C P}(1), \Omega)$

$\pi$ :projection on function that are constant on circles of latitudes $=$ zero Fourier coefficient .

Now let us prove the theorem. Let $g \in \mathcal{O}_{f}$. Let $\mu^{m}=\left(\mu_{k}^{m}\right)_{k}$ be the eigenvalues of the Hermitian matrix $T_{g}^{m}=\left(T_{g}^{m}\right)_{k, l}$. Schur's Theorem for Hermitian matrix gives

$$
\left(T_{g}^{m}\right)_{k, k} \prec \mu^{m} \text {. }
$$

This translated to step functions becomes as in (??)

$$
S^{m}\left(T_{g}^{m}\right)_{k, k} \prec S^{m}\left(\mu^{m}\right)
$$

Taking the limit as $\mathrm{m}$ goes to infinity we get

$$
\lim _{m \longrightarrow \infty} S^{m}\left(T_{g}^{m}\right)_{k, k}=\frac{1}{2 \pi} \int_{0}^{2 \pi} g(r, \theta) d \theta \prec \lim _{m \longrightarrow \infty} S^{m}\left(\mu^{m}\right)=g^{*}=f^{*} .
$$

To prove the converse, let us recall the following Theorem in ([ [?],p 523]) which states that the set $\Omega(f)=\{g \mid g \prec f\}$ is weakly compact and convex and its extreme points are precisely the points $f^{*} \circ \phi$, where $\phi$ is a measure preserving transformation of the unit interval. It is also shown in [ [?], p1030] that the set of extreme points is dense in $\Omega\left(f^{*}\right)$ for the weak topology. Combining these 
with our previous result that $L s \sum_{m} \cdot \Lambda^{m}$ is the set $f^{*} \circ \phi$, it comes out that $\Omega\left(f^{*}\right)$ is the weak closure of $L s \sum_{m} \cdot \Lambda^{m}$.

$$
\Omega\left(f^{*}\right)={\overline{L s \sum_{m} \cdot \Lambda^{m}}}^{\text {weak }} .
$$

The proof of the converse is based on the following diagram

$$
\begin{aligned}
& \widetilde{G} \in L^{2}(\mathbb{C P}(1)) \stackrel{\pi}{\longrightarrow} g \in \Omega\left(f^{*}\right)=\frac{\downarrow_{\text {weaklimit }}}{\left.\downarrow^{T^{m}}\left(\sum_{m} \cdot \Lambda^{m}\right)\right)} \text { weak } \\
& G_{\alpha} \in \operatorname{su}(m+1) \stackrel{\pi_{m}}{\longrightarrow} \quad\left(g_{\alpha}\right) \in \operatorname{co}\left(\sum_{m}\left(\Lambda^{m}\right)\right)
\end{aligned}
$$

For $g \in \Omega(f)=\overline{L s \sum_{m} \cdot \Lambda^{m}}{ }^{\text {weak }}$, let $g_{\alpha} \in L s \sum_{m_{\alpha}} \cdot \Lambda^{m_{\alpha}}$ be a generalized sequence that converges weakly to $g$.

A theorem by Rado states $g_{\alpha} \in c o\left(\sum_{m_{\alpha}} \cdot \Lambda^{m_{\alpha}}\right) \Leftrightarrow g_{\alpha} \prec \Lambda^{m_{\alpha}}$. Horn's Theorem implies that there exists a Hermitian matrix $G_{\alpha}$ whose spectrum is $\lambda^{m_{\alpha}}$ and its principal diagonal is $g_{\alpha}$. Now since Toeplitz quantization is surjective, there exists a function $\widetilde{G_{\alpha}}$ such that $T_{\widetilde{G_{\alpha}}}^{m_{\alpha}}=G_{\alpha}$.

As before we have

$$
\operatorname{tr}\left(T_{\widetilde{G_{\alpha}}}^{m_{\alpha}}\right)^{p}=\frac{\sum_{k=0}^{k=m_{\alpha}}\left(\lambda_{k}^{m_{\alpha}}\right)^{p}}{m_{\alpha}+1}
$$

Therefore

$$
\lim _{m_{\alpha} \longrightarrow \infty} \frac{\sum_{k=0}^{k=m_{\alpha}} \phi\left(\lambda_{k}^{m_{\alpha}}\right)}{m_{\alpha}+1}=\frac{1}{2 \pi} \int_{\mathbb{C P}(1)} \phi\left(\widetilde{G_{\alpha}}\right) d \Omega
$$

Which because of equality (??) gives

$$
{\widetilde{G_{\alpha}}}^{*}=f^{*}
$$

So for large $m_{\alpha}$, the functions $\widetilde{G_{\alpha}}$ are in the orbit of $f$ which is weakly compact

Therefore we can extract a sub-generalized sequence that we denote again by $\widetilde{G_{\alpha}}$ which converges weakly to a function $\widetilde{G}$ which because of the weak compactness belongs to the orbit of $f$.

To summarize, we have constructed a generalized sequence $\left(\widetilde{G_{\alpha}}\right)_{\alpha} \in \mathcal{O}(f)$ that converges weakly to $\widetilde{G}$ such that the Toeplitz quantization $T_{\widetilde{G_{\alpha}}}^{m_{\alpha}}$ has spectrum $\lambda^{m_{\alpha}}$ and principal diagonal $g_{\alpha}$.

The projection $\pi_{m}$ still converge to $\pi$ for the weak topology.

$$
\begin{aligned}
\pi(\widetilde{G}) & =\lim _{\alpha} \pi\left(\widetilde{G_{\alpha}}\right) \\
& =\lim _{m_{\alpha}} \pi_{m_{\alpha}}\left(g_{\alpha}\right) \\
& =g
\end{aligned}
$$

and that ends the proof of the Theorem. 
Based on this result, we strongly believe that Toeplitz Quantization can be used to extend many results from finite dimensional Lie group and Lie algebra to infinite dimensional group of symplectomorphisms of compact manifold.

\section{Acknowledgment}

I would like thank my advisor professor H. Flaschka for his valuable discussions and comments.

This project was supported by King Khaled University under grant KKU $\mathrm{S}_{\mathrm{S}} 277_{33}$.

\section{References}

[1] C. D. Aliprantis, K. C. Border, Infinite Dimensional Analysis, a Hitchhiker's Guide, 3rd edition, Springer (2005).

[2] A.Bloch, H.Flaschka, T.Ratiu, A Shur-Horn-Kostant Convexity Theorem for the Diffeomorphism Group of the Annulus, Inv.Math. 113, 511-529, (1993).

[3] A. Bloch, M. El Hadrami, H. Flaschka, T. Ratiu, Maximal Tori Of Some Symplectomorphism Groups And Applications To Convexity, Proceedings of Ascona Meeting, June 1996. (D. Sternheimer, J. Rawnsley, S. Gutt, eds.), Mathematical Physics Studies 20, Kluwer Academic Publishers, 201-222, (1997).

[4] M. Bordemann, E. Meinrenken and M. Schlichenmaier, Toeplitz Quantization Of Kähler Manifolds And $g l(N), N \rightarrow+\infty$ Limits., Commun. Math. Phys. 165 , 281-296,(1994).

[5] J. R. Brown, Approximation theorems for Markov Operators, Pac.J.Maths.16, 13-23, (1966).

[6] N. Dunford and J . T. Schwartz, Linear Operators, Part I, New York, 1958.

[7] Richard Durrett Probability: Theory And Examples Wadsworth \& brooks/cole Advanced Books

[8] Victor Guillemin [1979] Some Classical Theorem In Spectral Theory Revisited, Seminar on Singulariries of Solutions of Linear Partial Differential Equations (L. Hörmander ed) Annals of Mathematics Studies, 91, Princeton University Press and University of Tokyo Press, 219- 259.

[9] Lucas Grafakos, Classical Fourier Analysis. Second Edition. Graduate Texts in Mathematics, 249, Springer Verlag, (2008).

[10] P. Halmos Approximation Theorems for Measure Preserving Transformations, Trans.AMS 55, 1-18, (1944). 
[11] B. Kostant, On convexity, the Weyl Group and the Iwasawa Decomposition, Ann.Sci.Ec.Norm.Sup 6, 413-455, (1973).

[12] M. M. Rao Measure Theory And Integration, 2nd Edition, Marcel Dekker Inc, (2004).

[13] Jean-Michel Rakotoson Réarrangement Relatif, Un Instrument d'Estimation dans les Problèmes aux Limites, Mathématiques \& Applications, 64, Springer, (2008).

[14] J. V. Ryff, On the Representation of Doubly Stochastic Operators, Pac.J.Maths.13, 1379-1386, (1963).

[15] J. V. Ryff, Orbits of $L^{1}$ Functions Under Doubly Stochastic Transformations, Trans.AMS 117, 92-100, (1965).

[16] J. V. Ryff, Extreme points of some convex subsets of $L^{1}(0,1)$, Proc. AMS $18,1026-1034,(1967)$. 